\title{
Modelling the atomic structure of very high-density amorphous ice
}

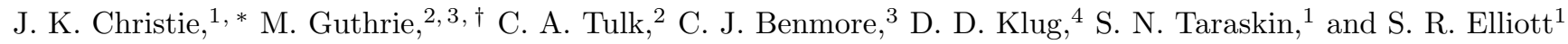 \\ ${ }^{1}$ Department of Chemistry, Cambridge University, Lensfield Road, Cambridge, CB2 1EW, UK \\ ${ }^{2}$ Spallation Neutron Source, Oak Ridge National Laboratory, Oak Ridge, TN 37831 \\ ${ }^{3}$ Argonne National Laboratory, Argonne, IL 60439 \\ ${ }^{4}$ National Research Council of Canada, Ottawa, Ontario, Canada KA1 OR6
}

(Dated: October 28, 2018)

\begin{abstract}
The structure of very high-density amorphous (VHDA) ice has been modelled by positionally disordering three crystalline phases, namely ice IV, VI and XII. These phases were chosen because only they are stable or metastable in the region of the ice phase diagram where VHDA ice is formed, and their densities are comparable to that of VHDA ice. An excellent fit to the medium range of the experimentally observed pair-correlation function $g(r)$ of VHDA ice was obtained by introducing disorder into the positions of the $\mathrm{H}_{2} \mathrm{O}$ molecules, as well as small amounts of molecular rotational disorder, disorder in the $\mathrm{O}-\mathrm{H}$ bond lengths and disorder in the $\mathrm{H}-\mathrm{O}-\mathrm{H}$ bond angles. The low- $k$ behaviour of the experimental structure factor, $S(k)$, is also very well reproduced by this disordered-crystal model. The fraction of each phase present in the best-fit disordered model is very close to that observed in the probable crystallization products of VHDA ice. In particular, only negligible amounts of ice IV are predicted, in accordance with experimental observation.
\end{abstract}

PACS numbers: $61.43 .-\mathrm{j}$

The study of water has attracted much attention [1, 2]. This is not only because of its importance in nature, but also because it has a number of unusual properties. In particular, it has a very rich phase diagram. In addition to liquid water, thirteen crystalline phases of ice are known to exist, as well as at least three amorphous, or glassy, phases [2]. Mishima et al. [3] were the first to synthesize high-density amorphous (HDA) ice, and the relationships between this phase, low-density amorphous (LDA) ice, and liquid water have been well studied [4]. Recently, another distinct phase of amorphous ice has been made, by heating HDA ice isobarically at $1-2 \mathrm{GPa}$ from $77 \mathrm{~K}$ to $\sim 165 \mathrm{~K}$. The new phase of ice can be recovered at $77 \mathrm{~K}$ and 1 bar 5]. This new phase is even higher in density than HDA ice, and has become known as very high-density amorphous (VHDA) ice [5]. VHDA ice, and its relationship to HDA and LDA ice, is of considerable interest in studies of polyamorphism [ [] (in which a substance exhibits more than one amorphous phase), and its structure is also relevant to the structure of liquid water.

Water molecules form strong hydrogen bonds to neighbouring water molecules, resulting in a roughly tetrahedral molecular arrangement. The hydrogen-bondinduced flexibility of this structure is the main reason for the many different phases of ice. HDA and VHDA ice exhibit closer packing of the water molecules. Each water molecule is still bonded to four nearest neighbours but, between the first and second neighbour shells, there is approximately one extra molecule in HDA ice, and two extra molecules in VHDA ice. This increase in coordination number is observed experimentally [7, 8, 9] and attributed to occupation of the "lynch pin" location by interstitial water molecules which are not hydrogen bonded to the origin molecule. Recent MD simulations support this interpretation [10], and suggest that VHDA ice is more stable than HDA ice, and that it is the amorphous analogue of the postulated high-density liquid water structure [1].

The medium-range structure of amorphous materials, that is how atomic positions are correlated over several interatomic distances, is of much interest 12]. VHDA ice is exceptional among amorphous materials in exhibiting very pronounced medium-range order (MRO). The orientationally averaged real-space pair-correlation function, $g(r)$, for $r \gtrsim 5 \AA$, consists of damped real-space oscillations extending to $\sim 20 \AA$, with only one significant period, $D$ 13. The orientationally averaged structure factor, $S(k)$, measured by neutron and X-ray diffraction [13], exhibits an intense, sharp peak at $k_{0}=2.29(2) \AA^{-1}$ in the neutron data, and $2.30(2) \AA^{-1}$ in the X-ray data, this peak being the dominant feature (particularly for the neutron data). Since $g(r)$ and $S(k)$ are related by a Fourier transform, the period of the oscillation in $g(r)$ is related to the position of the peak in $S(k)$ by $D=2 \pi / k_{0}$.

The structure of amorphous materials has recently been modelled by adding Gaussian positional disorder into the atomic structure of crystalline phases of these materials [14, 15]. These disordered crystals gave good representations of the MRO of some amorphous materials, but not necessarily the short-range order (SRO). In particular, the value of the wavevector transfer of the so-called first sharp diffraction peak (FSDP, a characteristic peak in the $S(k)$ of many amorphous materials at low wavevector, $k \sim 1-2 \AA^{-1}$ ) was successfully predicted in amorphous silicon and vitreous silica. The existence of the FSDP is often regarded as a signature of MRO [12].

For a model crystalline lattice, $S(k)$ consists of a series of $\delta$-functions caused by scattering from the various Bragg planes. The effect of Gaussian positional disorder is to multiply these peaks by the Debye-Waller factor, 
$e^{-k^{2} \sigma^{2}}$, where $\sigma$ is the half width of the Gaussian distribution, and to produce a low-amplitude diffuse scattering 16. It is clear that the high- $k$ peaks will be destroyed first by introducing disorder, and that for large enough disorder (high enough $\sigma$ ), only one peak (that at the lowest value of $k$ ) will contribute substantially to $S(k)$.

Similarly, for a model crystalline lattice, $g(r)$ consists of $\delta$-functions at the various interatomic distances. The effect of Gaussian positional disorder is to convolve these peaks with the Fourier transform of the Debye-Waller factor, which is also a Gaussian [16]. For large enough disorder (high enough $\sigma$ ), these Gaussian peaks broaden and combine to form damped oscillations in $g(r)$ with a single period [14, 15. This functional behaviour, of course, results from the Fourier transformation of $S(k)$ having only one significant peak.

The experimentally measured $g(r)$ for VHDA ice 13], showing pronounced MRO, characterized by nearly periodic oscillations extending to large $r$, accompanied by a dominant sharp low- $k$ peak in $S(k)$, are very reminiscent of the simulated data for positionally disordered lattices [14, 15]. It was natural to ask whether the MRO in VHDA ice could be modelled by disordering crystalline ice. The structure of an amorphous phase is most likely to resemble the crystalline phase with a similar density that is stable at similar temperatures and pressures. VHDA ice is formed at $\sim 165 \mathrm{~K}$ and $1-2 \mathrm{GPa}$, with density $\rho=1.25 \mathrm{~g} / \mathrm{cm}^{3}$ (all densities are for $\mathrm{H}_{2} \mathrm{O}$ ) when recovered to 1 bar at $77 \mathrm{~K}[5]$. According to the phase diagram, ice VI is the only crystalline phase stable in this region [2]. Four other crystalline phases $\left(\mathrm{I}_{c}, \mathrm{IV}, \mathrm{IX}\right.$ and XII) are metastable 2] and hence their occurrence around these temperatures and pressures cannot in principle be excluded. However, ice $\mathrm{I}_{c}$ and IX can be eliminated as candidates because their densities (respectively $0.93 \mathrm{~g} / \mathrm{cm}^{3}$ at $77 \mathrm{~K}$ and $1 \mathrm{bar}\left[2\right.$, and $1.17 \mathrm{~g} / \mathrm{cm}^{3}$ at $110 \mathrm{~K}$ and 1 bar 17]) are so much lower than that of VHDA ice, and it is very unlikely that a positionally disordered phase could have a larger density than the corresponding crystalline phase. If there were significant density variations in VHDA ice, then small-angle scattering would be experimentally observed, which it is not. It was therefore decided to disorder the structures of ice IV $(\rho=1.29$ $\mathrm{g} / \mathrm{cm}^{3}$ at $260 \mathrm{~K}$ and $\left.0.5 \mathrm{GPa}[18]\right), \mathrm{VI}\left(\rho=1.37 \mathrm{~g} / \mathrm{cm}^{3}\right.$ at $225 \mathrm{~K}$ and $1.1 \mathrm{GPa} 20)$ and XII $\left(\rho=1.29 \mathrm{~g} / \mathrm{cm}^{3}\right.$ at $260 \mathrm{~K}$ and $0.5 \mathrm{GPa}[18]$ ), in order to try to simulate VHDA ice.

Crystal models (with $\sim 3 \times 10^{4}$ atoms) of each phase were created. The crystalline atomic positions for ice IV [19], VI 20] and XII [19] were taken from neutrondiffraction data. The hydrogen atoms were positioned according to the "ice rules" 21]: along each $\mathrm{O}-\mathrm{O}$ bond there is only one hydrogen atom, which is covalently bonded (and thus closer) to one oxygen atom, and hydrogen bonded to (and thus further away from) the other oxygen atom. For these phases, this means that only

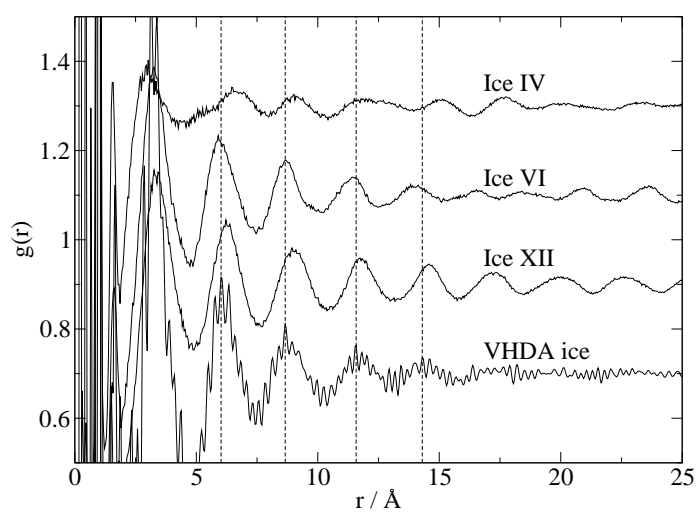

FIG. 1: The computed pair-correlation function, $g(r)$, for positionally disordered ice IV, VI and XII lattices, compared to experimental data [13] for VHDA ice. The parameters used to generate the simulated curves are those of the best-fit threephase models, as given in Table【 The dashed vertical lines show the peak positions in $g(r)$ for VHDA ice. It is clear that the peak positions and periods of oscillation are different for each phase. The $g(r)$ 's have been offset vertically for clarity.

half of the sites available for hydrogen atoms are occupied; this fractional occupancy is observed experimentally [19, 20]. An algorithm due to Buch et al. 22] was used to position the hydrogen atoms on their crystalline sites. The models were rescaled slightly so that their densities matched that of VHDA ice.

Disorder was then introduced into the models as follows. Positional disorder was introduced by displacing the $\mathrm{H}_{2} \mathrm{O}$ molecules rigidly in the $x-, y$ - and $z$-directions. Then the $\mathrm{H}_{2} \mathrm{O}$ molecules were rotated around the $x$-, $y$ and $z$-axes passing through the oxygen atom positions, such that the oxygen atoms were kept stationary. The hydrogen atoms were then moved along the $\mathrm{O}-\mathrm{H}$ bond direction, to disorder the $\mathrm{O}-\mathrm{H}$ bond lengths. Finally, the hydrogen atoms were symmetrically moved in the $\mathrm{H}-\mathrm{O}-$ $\mathrm{H}$ plane to disorder the $\mathrm{H}-\mathrm{O}-\mathrm{H}$ bond angles. In each case, the change in the relevant quantity (position or angle) was randomly chosen from a Gaussian distribution centred on zero, with half widths as given in Table \

Calculated $g(r)$ 's for these models were fitted to neutron-diffraction data 13] of deuterated VHDA ice, the structure of which differs negligibly from the structure of the hydrogenated material [23]. The neutron data were used, as they were of a higher quality than the X-ray data. Since particularly good agreement with the data at low $r$ was not expected, the data for $r<7 \AA$ were excluded from the fit. The partial pair-correlation functions $g_{O O}(r), g_{O D}(r)$ and $g_{D D}(r)$ of our models were combined to give the full neutron $g(r)$ as $g(r)=0.088 g_{O O}(r)+$ $0.418 g_{O D}(r)+0.494 g_{D D}(r)$, as in Ref. [13].

The calculated $g(r)$ 's for the positionally disordered models of each separate phase do not exactly fit the $g(r)$ 


\begin{tabular}{|c|c|c|c|c|c|c|c|}
\hline & Half width & Range studied & $\begin{array}{c}\text { ice IV } \\
3 \text { phases }\end{array}$ & $\begin{array}{r}\text { ice } \\
2 \text { phases } \\
\end{array}$ & $\begin{array}{l}\text { VI } \\
3 \text { phases }\end{array}$ & $\begin{array}{r}\text { ice } \\
2 \text { phases }\end{array}$ & $\begin{array}{l}\text { XII } \\
3 \text { phases }\end{array}$ \\
\hline Positional disorder & $\sigma_{p o s}$ & $0.05-0.30 \AA$ & $0.22 \AA$ & $0.16 \AA$ & $0.16 \ddot{A}$ & $0.19 \AA$ & $0.18 \ddot{A}$ \\
\hline Rotational disorder & $\sigma_{\theta}$ & $0.01-0.05 \mathrm{rad}$ & $0.04 \mathrm{rad}$ & $0.04 \mathrm{rad}$ & $0.04 \mathrm{rad}$ & $0.03 \mathrm{rad}$ & $0.01 \mathrm{rad}$ \\
\hline $\mathrm{O}-\mathrm{H}$ bond length disorder & $\sigma_{O H}$ & $0.01-0.04 \AA$ & $0.03 \AA$ & $0.04 \AA$ & $0.04 \AA \vec{A}$ & $0.02 \AA$ & $0.01 \AA ̊ A$ \\
\hline $\mathrm{H}-\mathrm{O}-\mathrm{H}$ bond angle disorder & $\sigma_{H O H}$ & $0.01-0.04 \mathrm{rad}$ & $0.01 \mathrm{rad}$ & $0.01 \mathrm{rad}$ & $0.01 \mathrm{rad}$ & $0.03 \mathrm{rad}$ & $0.02 \mathrm{rad}$ \\
\hline Volume fraction, $f$ & - & $0-1$ & 0.035 & 0.544 & 0.549 & 0.456 & 0.416 \\
\hline
\end{tabular}

TABLE I: Disorder parameters for the crystalline phases, ice IV, VI and XII. The ranges of values of the disorder parameters and the fractions of each phase, and the values in the best-fit two- and three-phase models, are also given. In each case, the change in the position or angle was randomly taken from a Gaussian distribution, centred on zero, with half widths as shown.

of VHDA ice (see Fig. 1).

Hence, the structure of VHDA ice was assumed to comprise an inhomogeneous mixture of regions of positionally disordered ice IV, VI and XII, of indeterminate size, but with total volume fractions of $f_{I V}, f_{V I}$ and $f_{X I I}=$ $1-\left(f_{I V}+f_{V I}\right)$, respectively. The $g(r)$ 's of the three phases were combined to give a total pair-correlation function: $g(r)=f_{I V} g_{I V}(r)+f_{V I} g_{V I}(r)+f_{X I I} g_{X I I}(r)$, where $0<f_{I V}, f_{V I}, f_{X I I}<1$, and e.g. $g_{I V}(r)$ is the full $g(r)$ of the disordered ice IV lattice. For each combination of the $g(r)$ 's of the three phases, a least-squares minimization was performed with respect to $f_{I V}$ and $f_{V I}$.

The minimization analysis showed that $\sigma_{p o s}$ and the volume fractions were the most significant parameters in determining the quality of the fits in the medium range $(r \geq 7 \AA)$. The other parameters, $\sigma_{\theta}, \sigma_{O H}$ and $\sigma_{H O H}$, were much less important, and only significantly affected $g(r)$ at low $r$. The best-fit model (with parameters as given in Table 【) gives a total $g(r)$ with medium-range oscillations in very good agreement with those found experimentally for VHDA ice (Fig. 2), and very significantly better than for the disordered individual phases (see Fig. 11). The short-range fit is less good; in particular, the heights of the peaks at $\sim 1 \AA$ and $\sim 3.3 \AA$, are not reproduced so well (see inset to Fig. 2). The height of the latter peak is known to be sample and pressure dependent, so one would not necessarily expect a good fit. The best-fit model has a least-squares deviation from the experimental data of 0.0777 . It contains $3.5 \%$ ice IV, an amount which is not resolvable by diffraction studies. The fitting procedure was repeated for disordered ice VI and XII only, and the best-fit model (Fig. (2) has a deviation of 0.0779 , which is only $0.25 \%$ more than the deviation for the best three-phase model. The corresponding $g(r)$ 's are indistinguishable (Fig. 2). We conclude that the amount of ice IV needed to model VHDA ice is negligible, and that disordering only ice VI and XII produces just as good a description of the MRO of VHDA ice.

It is also instructive to compare the structure factor $S(k)$ of the best-fit model (calculated by Fourierinverting $g(r)$ ) with the experimental data (Fig. 3). The height, width and position of the first peak are fitted very well, which is expected since this peak governs the medium-range real-space behaviour. The fit at higher $k$

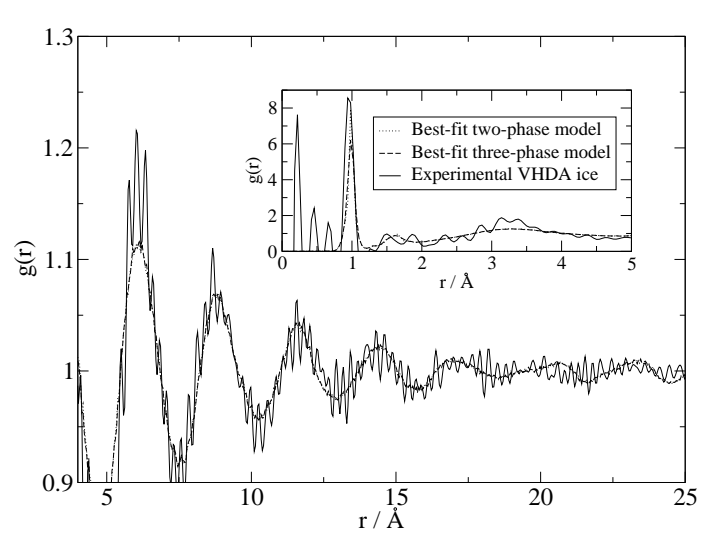

FIG. 2: The pair-correlation functions $g(r)$ of the best-fit models consisting of disordered lattices of ice IV, VI and XII (dashed line) and ice VI and XII only (dotted line), compared with the experimentally measured neutron-diffraction $g(r)$ of VHDA ice [13] (solid line). The disorder parameters of the models are given in Table \ The best-fit two- and three-phase models are indistinguishable for medium-range distances. The $g(r)$ for VHDA ice was calculated by Fourier inversion of the experimentally measured structure factor 13. without smoothing, resulting in considerable noise, and spurious peaks for $r \lesssim 0.8 \AA$.

(which corresponds to small $r$ ) is less good, although the peaks are reasonably well reproduced.

When Klotz et al. recrystallized high-pressure HDA (probably VHDA) ice, the fractions of the phases present were $56 \%$ ice VI and $44 \%$ ice XII 19. These are very close to the simulated values of $54 \%$ ice VI and $46 \%$ ice XII for the best-fit two-phase model. This implies that the structure of VHDA ice is intimately related to the structures of ice VI and XII. It is also interesting to note that the value of $\sigma_{p o s}$ in our models (Table \) is comparable to the thermal mean-square atomic displacement, which for these phases is $\sim 0.2 \AA[19,20]$.

Attempts were made to improve the fit at short range, by fitting separately to the low- $r$ region of $g(r)$, and to the medium- and high- $k$ region in $S(k)$, whilst varying the short-range disorder parameters $\sigma_{\theta}, \sigma_{\mathrm{OH}}, \sigma_{\mathrm{HOH}}$ and volume fractions, but none of these attempts improved the fit of the model. Some of this discrepancy may be 


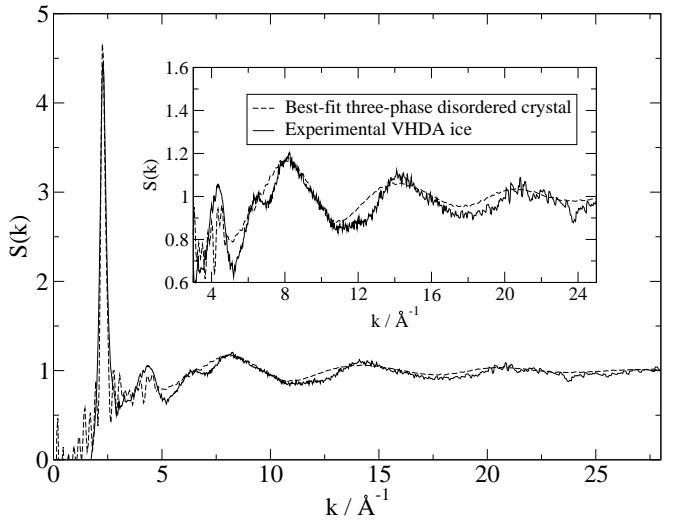

FIG. 3: The structure factor $S(k)$ of the best-fit model consisting of disordered lattices of ice IV, VI and XII, compared with the experimentally measured neutron-diffraction $S(k)$ of VHDA ice [13]. The parameters of the model are given in Table【

attributed to effects at the boundaries between regions of disordered ice phases proposed in the VHDA ice structure. Our models take no account of these boundaries, yet if the ice structure consists of positionally disordered domains of different crystal phases, then the interfacial regions will not necessarily resemble any of the phases. It is likely that any such distortion will be fairly local, extending over maybe two nearest-neighbour bond lengths, confining these surface effects to low $r$.

The fact that the disordered crystal model is able to reproduce the medium-range structure, but not particularly that of the first- and second-peak region in $g(r)$, for VHDA ice may be an important indication of structural differences between the amorphous and crystalline phases. It is well known that the 4-8 $\AA$ range in amorphous ice is very sensitive to changes in pressure, and Xray diffraction studies of VHDA ice have shown that this region contains two peaks which are most likely related to the start of the formation of non-bonded interpenetrating lattices (similar to those present in ice VI and XII). A distortion of the local tetrahedral unit in VHDA ice enables an increased packing of interstitial molecules, suggesting that much of the pressure-induced frustration in the material occurs largely in the ordering of the molecules just beyond the first shell. This frustration arises from competition between the optimum local packing of molecules and the preferred longer-range topology of the network. Such high-density structures are very different from that characterized by the well-defined first two shells found in LDA ice, which closely resembles that of ice $I_{h}$.

In conclusion, the medium-range structure of very high-density amorphous (VHDA) ice has been modelled by adding positional disorder to three crystalline phases of ice, namely ice IV, ice VI and ice XII. These phases were chosen because they are stable or metastable in the region of the phase diagram where VHDA ice is formed, and their densities are similar to that of VHDA ice. For medium-range distances $(r \gtrsim 7 \AA)$, the fit to the experimentally observed neutron-diffraction $g(r)$ is very good. The fractions of each phase present in the model which gives the best fit to the experimental data are very close to those found when a sample, which was likely to have been VHDA ice, was recrystallized [19]; in particular, the amount of ice IV present is negligible (none was observed experimentally). We conclude that the structure of VHDA ice at large enough distances from an arbitrary origin atom can be well modelled as an inhomogeneous mixture of positionally disordered ice VI and ice XII.

JKC would like to thank the Engineering and Physical Sciences Research Council for financial support.

* Electronic address: jkc25@cam.ac.uk

† Current affiliation: Centre for Science at Extreme Conditions, Edinburgh University, Edinburgh, EH9 3JZ, UK

[1] Water: A comprehensive treatise, ed. F. Franks, Plenum Press (New York, 1972).

[2] Physics of Ice, V. F. Petrenko and R. W. Whitworth, Oxford University Press (Oxford, 1999).

[3] O. Mishima, L. D. Calvert and E. Whalley, Nature 310, 393 (1984); Ibid. 314, 76 (1985).

[4] Metastable Liquids, P. G. Debenedetti, Princeton University Press (Princeton, 1996).

[5] T. Loerting, C. Salzmann, I. Kohl, E. Mayer and A. Hallbrucker, Phys. Chem. Chem. Phys. 3, 5355 (2001).

[6] C. A. Angell, Annu. Rev. Phys. Chem. 55, 559 (2004).

[7] A. K. Soper, Chem. Phys. 202, 295 (1996).

[8] J. L. Finney, D. T. Bowron, A. K. Soper, T. Loerting, E. Mayer and A. Hallbruker, Phys. Rev. Lett. 89, 205503 (2002).

[9] J. L. Finney, A. Hallbruker, I. Kohl, A. K. Soper, and D. T. Bowron, Phys. Rev. Lett. 88, 225503 (2002).

[10] N. Giovambattista, H. E. Stanley and F. Sciortino, cond-mat/0502531

[11] O. Mishima and H. E. Stanley, Nature 396, 329 (1998).

[12] S. R. Elliott, Nature 354, 445 (1991).

[13] M. Guthrie, C. A. Tulk, C. J. Benmore and D. D. Klug, Chem. Phys. Lett. 397, 335 (2004).

[14] J. K. Christie, S. N. Taraskin and S. R. Elliott, Phys. Rev. B 70, 134207 (2004).

[15] J. K. Christie, S. N. Taraskin and S. R. Elliott, J. Phys.: Condens. Matter 16, S5109 (2004).

[16] B. T. M. Willis and A. W. Pryor, Thermal Vibrations in Crystallography, Cambridge University Press, (Cambridge, 1975).

[17] J. D. Londono, W. F. Kuhs and J. L. Finney, J. Chem. Phys. 98, 4878 (1993).

[18] C. Lobban, J. L. Finney and W. F. Kuhs, Nature 391, 268 (1998).

[19] S. Klotz, G. Hamel, J. S. Loveday, R. J. Nelmes and M. Guthrie, Z. Kristallogr. 218, 117 (2003).

[20] W. F. Kuhs, J. L. Finney, C. Vettier and D. V. Bliss, J. Chem. Phys. 81, 3612 (1984).

[21] L. Pauling, J. Am. Chem. Soc. 57, 2680 (1935). 
[22] V. Buch, P. Sandler and J. Sadlej, J. Phys. Chem. B 102, 8641 (1998).

[23] B. Tomberli, C. J. Benmore, P. A. Egelstaff, J. Neuefeind and V. Honkimaki, J. Phys.: Condens. Matter 12, 2597 (2000). 